\title{
Efecto de la alta temperatura en la germinación y supervivencia de semillas de la especie invasora Pinus contorta y dos especies nativas del sur de Chile
}

\author{
Effects of high temperatures in the germination and seed survival of the invasive \\ species Pinus contorta and two native species of South Chile
}

\author{
Ana J Cóbar-Carranza a,b*, Rafael A García b , Aníbal Pauchard a,b, Eduardo Peña a \\ *Autor de correspondencia: ${ }^{a}$ Universidad de Concepción, Facultad de Ciencias Forestales, Laboratorio Invasiones Biológicas, \\ Victoria 631, Barrio Universitario, Concepción, Chile, tel.: +56 -041-2661132, anacobar@gmail.com

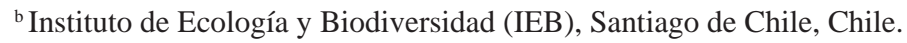

\begin{abstract}
SUMMARY
Studies in South America have reported increments of invasivity of Pinus spp. after fire events. These increments are due to morphological and physiological adaptations to wildfires that some species of this specific genus presents. Pinus contorta is an invasive species in temperate ecosystems, affecting Araucaria araucana and Nothofagus antarctica forests in Malalcahuello National Reserve, Chile. In order to comprehend the regeneration response to post-fire, it is necessary to know the response and tolerance of the seeds to high temperatures. Seeds of $P$. contorta, A. araucana and $N$. antarctica were sown in pots with sand and subsequently heated at 80,100 and $120^{\circ} \mathrm{C}$ for five minutes. Germination and seedling survival tests were carried out in a greenhouse. Germination in N. antarctica was null. Heat treatments did not have a negative effect on the germination of $P$. contorta, but they did on $A$. araucana inhibiting the germination at temperatures over $100{ }^{\circ} \mathrm{C}$. These results suggest that when undergoing a fire of high intensity, $P$. contorta has a competitive advantage over A. araucana and N. antarctica; the seed bank would have more possibilities to survive the effects of a wildfire.
\end{abstract}

Key words: Araucaria araucana, Nothofagus antarctica, wildfire, fire ecology, heat shock.

\section{RESUMEN}

Estudios en Sudamérica han reportado aumentos en la invasividad de Pinus spp. después de eventos de fuego. Esto se debe a adaptaciones morfológicas y fisiológicas a incendios que presentan algunas especies de este género. Pinus contorta es uno de los pinos más invasores en ecosistemas templados y se encuentra invadiendo los bosques de Araucaria araucana y Nothofagus antarctica en la Reserva Nacional Malalcahuello, Chile. Para comprender la respuesta potencial de la regeneración post-incendios en árboles, es necesario conocer la respuesta y tolerancia de la semilla a altas temperaturas. Semillas de P. contorta, A. araucana y $N$. antarctica fueron sembradas en bandejas con arena y sometidas a 80,100 y $120^{\circ} \mathrm{C}$ por cinco minutos, después fueron llevadas al invernadero para evaluar la germinación y supervivencia de las plántulas. La germinación para $N$. antarctica fue nula incluso en el control. Las altas temperaturas no presentaron efectos negativos en la germinación de $P$. contorta, pero sí en A. araucana inhibiendo la germinación a temperaturas mayores a $100^{\circ} \mathrm{C}$. Estos resultados sugieren que, en incendios de alta intensidad, las semillas de $P$. contorta tiene una ventaja competitiva sobre $A$. araucana y N. antarctica, ya que el banco de semillas tendrá mayores posibilidades de sobrevivir ante un incendio forestal.

Palabras clave: Araucaria araucana, Nothofagus antarctica, incendio forestal, ecología del fuego, choque térmico.

\section{INTRODUCCIÓN}

Los incendios forestales afectan las dinámicas poblacionales de las plantas y favorecen las invasiones biológicas (Richardson et al. 1994). Este fenómeno se ha reportado en el hemisferio sur en ecosistemas invadidos por diferentes especies de Pinaceae, observándose que tras incendios forestales la regeneración de individuos de Pinus spp. aumenta (e.g. Pinus ponderosa Douglas ex. C. Lawson y Pinus halepensis Mill.) (Núñez y Raffaele 2007, Zalba et al. 2008). El éxito de establecimiento del género Pinus tras incendios forestales se debe a que algunas especies (e.g.
Pinus contorta Douglas ex Loudon) están adaptadas a incendios que provocan la destrucción de rodales forestales; y la recuperación de sus poblaciones depende del banco de semillas (Ledgard 2001). La formación de banco de semillas permanentes en el suelo en varias especies de Pinus es poco frecuente (Teste et al. 2011). Sin embargo, especies de pinos serótinos como $P$. contorta generan bancos de semillas viables en la copa o enterrados en el suelo (Turner et al. 2007, Teste et al. 2011). Se han reportado casos de banco de semillas almacenado en conos viejos que mantienen una viabilidad y vigor de la semilla aún después de 10 años de haber madurado el cono (Teste et al. 2011). El cono brinda 
protección a las semillas en los incendios soportando temperaturas de hasta $400{ }^{\circ} \mathrm{C}$, manteniendo aún viable la semilla (Habrouk et al. 1999), y la protege de la descomposición en el suelo ayudando a formar un banco de propágulos (Teste et al. 2011).

Dentro de los múltiples impactos que generan los incendios forestales sobre el ecosistema, destacan los efectos sobre las propiedades físicas, químicas, mineralógicas y biológicas del suelo. Se han reportado modificaciones como cambios en el pH, disponibilidad de nutrientes, porosidad, estabilidad de los agregados, repelencia al agua y disminución de la materia orgánica producto de los incendios forestales (DeBano et al. 1998). Sin embargo, estos cambios dependen de factores como la cantidad, humedad del combustible, la temperatura que alcanza en la superficie y la latencia de la combustión, los cuales definen la intensidad y duración del fuego (DeBano et al. 1998). Por lo tanto, la intensidad del incendio y la distribución del combustible en el suelo van a determinar el éxito de la germinación y los patrones de regeneración y establecimiento de plántulas el ambiente post-fuego (DeBano et al. 1998, Odion y Davis 2000, Herrero et al. 2007).

Los productos del fuego como el calor, el humo, las cenizas y madera carbonizada tienen una influencia física y fisiológica en la dormancia de semillas (Herrero et al. 2007). La germinación de semillas de muchas especies creciendo en ecosistemas dependientes del fuego, se ve favorecida por una breve exposición a altas temperaturas. Esto ocurre principalmente en especies de semillas pequeñas almacenadas en el banco de semillas (Odion y Davis 2000). La germinación es estimulada por choques térmicos entre 60 y $120^{\circ} \mathrm{C}$ aplicados entre $1-10$ minutos; temperaturas por arriba de $150{ }^{\circ} \mathrm{C}$ son letales para la mayoría de las especies (Habrouk et al. 1999, Herrero et al. 2007). Muchos estudios se han realizado sobre el efecto de altas temperaturas en la germinación (Habrouk et al. 1999, Herrero et al. 2007). Varios de estos estudios son con el género Pinus, donde se demuestra que para muchas especies la germinación disminuye con la temperatura y tiempo de exposición al calor, con variaciones en la tolerancia a las altas temperaturas según la especie (Habrouk et al. 1999, Herrero et al. 2007).

El efecto del fuego en el banco de semillas y la respuesta de la germinación de estas a diferentes rangos de temperatura del fuego, es clave para la recuperación de la vegetación. Es importante tener conocimiento sobre los mecanismos de germinación de las especies después de eventos de fuego, ya que esto permitirá comprender la respuesta del banco de semilla, el potencial reclutamiento y regeneración post-incendio; además de ser importante para la conservación de las especies nativas y el manejo de especies introducidas.

Pinus contorta es una especie altamente invasora que puede cambiar las propiedades del combustible afectando el comportamiento de los incendios forestales y los regímenes de fuego (Cóbar-Carranza et al. 2014). En los bosques de la Patagonia, esta especie alcanza una alta densidad, aumenta la carga de combustible y crea bosques en ecosistemas donde naturalmente los árboles están ausentes o en muy baja densidad (Langdon et al. 2010). Sin embargo, poco se sabe del efecto potencial del fuego en la viabilidad, germinación y supervivencia de las plántulas de $P$. contorta en ambientes invadidos. Sí el fuego afecta positivamente a $P$. contorta en comparación a las especies arbóreas nativas, se estaría en presencia de un indicador más de retroalimentación positiva entre fuego e invasión de pinos.

Pinus contorta se ha reportado invadiendo bosques nativos en el sur de Chile, como la Reserva Nacional Malalcahuello, donde en un período de 33 años ha invadido más de 78 ha de bosque nativo de las especies endémicas Araucaria araucana (Molina) K. Koch y Nothofagus antarctica (G. Forster) Oerst (Peña et al. 2008). El conocer la tolerancia a las altas temperaturas de las semillas de la especie invasora en comparación a las dos especies nativas, permitirá inferir sobre el potencial de regeneración tras un evento de incendio. Por lo tanto, el objetivo de este estudio es evaluar el efecto de las altas temperaturas en la germinación y supervivencia de plántulas de $P$. contorta, A. araucana y N. antarctica, con el fin de responder: ¿Cuáles son las diferencias en la capacidad germinativa de las semillas y supervivencia de plántulas a la aplicación de altas temperaturas de una especie invasora en comparación a dos especies nativas?

\section{MÉTODOS}

Especies de estudio. Pinus contorta es un árbol mediano (ca. $35 \mathrm{~m}$ de altura y $60 \mathrm{~cm}$ de DAP), intolerante a la sombra, pionero y de rápido crecimiento, que no presenta auto-poda, con una corteza de espesor medio de $5 \mathrm{~cm}$, que crece en áreas con altas limitantes nutricionales (Ledgard 2001). Su distribución nativa abarca regiones del oeste de Estados Unidos y Canadá, desde el nivel del mar hasta los 3.660 m s.n.m (Klinka 2002). Especie con gran amplitud ecológica y tolerancia, habita de oeste a este desde la región del Pacífico hasta la región Cordillerana, y desde la latitud $64^{\circ} \mathrm{N}$ en Yukon (Canadá) hasta la latitud $31^{\circ} \mathrm{N}$ en Baja California (Klinka 2002).

La especie produce semillas viables a temprana edad (5-10 años) (Peña et al. 2008). Cuenta con conos serótinos, pero esta característica es variable con la edad del árbol. Los árboles jóvenes tienen un mayor porcentaje de conos abiertos y los conos serótinos aumentan con la edad a partir de los 17 años (Ledgard 2001). La producción de conos es anual y produce abundantes semillas que fluctúan entre 500.000 a 800.000 semillas por hectárea por año (Klinka 2002). Las semillas tienen una viabilidad entre $65-90 \%$ y se dispersan por viento (Ledgard 2001). Las temperaturas óptimas para su germinación son entre $21-27^{\circ} \mathrm{C}$, siendo baja con temperaturas por debajo de los $15^{\circ} \mathrm{C}$ (Bates 1930). En su rango de distribución natural, es una especie 
adaptada a regímenes de incendios variables de fuegos superficiales de baja intensidad a fuegos de copa de alta intensidad (Ledgard 2001).

Araucaria araucana es una especie endémica de los bosques templados de Sudamérica. Se distribuye en la Cordillera de Los Andes (Chile y Argentina), y en la Cordillera de Nahuelbuta. Se desarrolla sobre un amplio rango ecológico, desde climas templado-cálido hasta climas con influencia mediterránea, tolerando temperaturas extremas desde $-20{ }^{\circ} \mathrm{C}$ hasta $30^{\circ} \mathrm{C}$ (González et al. 2013).

Árbol siempreverde, dioico, alcanza hasta $40 \mathrm{~m}$ de altura. En su forma adulta la copa es reducida y ramificada, corteza gruesa (20 cm de espesor) y sus hojas son coriáceas (González et al. 2013).

Araucaria araucana inicia la producción de flores y frutos a edades sobre los 15-25 años (González et al. 2013) y presenta una fluctuación anual en la producción de conos y semillas por árbol (promedio: 2 a 45 conos por árbol y 58 a 135 semillas por cono) (Sanguinetti y Kitzberger 2009). La viabilidad germinativa esta reportada entre 6080 \% (González et al. 2013). Las semillas están sometidas a depredación por la cachaña (Enicognathus ferrugineus Müller), que consume del 2-21 \% de semillas del árbol, y roedores que consumen 30 a $70 \%$ de semillas que caen al suelo; en tanto que la dispersión de semillas promedio del árbol madre es de 13 m (Sanguinetti y Kitzberger 2009). La fructificación y diseminación de semillas ocurre entre febrero-mayo y germinan en la primavera después del deshielo del invierno (González et al. 2013).

Los bosques de A. araucana están asociados a eventos de disturbio; adaptados a regímenes de incendios superficiales principalmente, aunque hay casos documentados de resistir incendios de copa, por su capacidad de rebrote epicórmico (González et al. 2013).

En 1990 en Chile A. araucana obtuvo el estatus de Monumento Natural, además se encuentra en el Apéndice I de CITES (González et al. 2013).

Nothofagus antarctica (G. Forster) Oerst. es una especie endémica de los bosques costeros templado lluvioso o subantárticos de Chile y Argentina, presentando la mayor amplitud ecológica de las especies de Nothofagus sudamericanos (Donoso et al. 2013). Esta especie adopta diferentes morfotipos dependiendo del ambiente en el que se desarrolla, y se sugiere que existen diferencias en las semillas según los morfotipos. El morfotipo arbóreo presenta semillas con mayor capacidad germinativa, en tanto los individuos achaparrados rara vez producen semillas, las cuales poseen escasa capacidad germinativa, y los caméfitos carecen de reproducción sexual (Donoso et al. 2013).

La especie florece entre octubre y noviembre y la fructificación se produce entre diciembre y abril. La producción de semillas reportada para la Patagonia Argentina es altamente variable, con promedio desde dos hasta $52 \mathrm{mi}-$ llones de semillas por hectárea (Bahamonde et al. 2011). En bosques nativos en Argentina se reportó que el establecimiento de plántulas corresponde al 0,5 \% del total de las semillas producidas y la supervivencia de plántulas al final del período de crecimiento fue igualmente baja (Bahamonde et al. 2011). Nothofagus antarctica es una especie adaptada a regímenes de incendios forestales, a pesar de quemarse completamente, sus raíces logran sobrevivir y rebrotar, favoreciendo la recuperación del ecosistema (Donoso et al. 2013).

Diseño del ensayo. Se utilizaron semillas de $P$. contorta colectadas de conos maduros de individuos adultos ubicados en la Reserva Nacional Malalcahuello en el año 2011 ( 87 \% germinación de semillas estratificadas, determinado en ensayo previo en cámara de germinación). Las semillas de $A$. araucana fueron adquiridas en la ciudad de Chillán y $N$. antarctica provenientes de la zona cordillerana en la provincia de Osorno. Las semillas fueron sometidas a estratificación por frío sobre arena húmeda a $4{ }^{\circ} \mathrm{C}$ por un período de 20 días para el caso de $P$. contorta y A. araucana, y 45 días para $N$. antarctica (Bates 1930, Stevens 1996, González et al. 2013).

Se evaluó el efecto de las altas temperaturas en las tres especies. Se probaron tres diferentes temperaturas 80, 100, $120^{\circ} \mathrm{C}$ por 5 minutos de exposición. Se seleccionaron estás temperaturas y exposición para simular los efectos de un incendio forestal (Habrouk et al. 1999, Herrero et al. 2007).

Para cada especie, se usaron cinco réplicas con 10 semillas para cada tratamiento, más cinco réplicas de semillas no expuestas a calor utilizadas como tratamiento control. El número de semillas seleccionado es similar a otros estudios donde utilizaron entre 15 y 20 semillas por réplica (e.g. Habrouk et al. 1999, Herrero et al. 2007). El calentamiento de las semillas se realizó en bandejas de aluminio con arena, para simular el efecto de las altas temperaturas generadas por un incendio sobre el banco edáfico de semillas.

Previo a calentar las semillas se colocó en el horno bandejas con arena, para asegurar que la arena alcanzara la temperatura deseada, la temperatura de la arena se determinó usando termocuplas tipo $\mathrm{K}$. Una vez alcanzada la temperatura deseada, las semillas de $P$. contorta y $N$. antarctica se sembraron a $1 \mathrm{~cm}$ de profundidad y las de A. araucana hasta quedar cubiertas por la arena. Luego las bandejas con las semillas fueron colocadas nuevamente en el horno a la temperatura deseada por 5 minutos. Después del tratamiento las bandejas se llevaron al invernadero, donde se mantuvieron a temperatura promedio de $27^{\circ} \mathrm{C}$. Las semillas se monitorearon cada dos días durante 123 días, y se regaron diariamente, tres veces al día por cinco minutos mediante riego aéreo automatizado por aspersión. Se consideró germinación cuando se observó emergencia del tallo. El experimento se llevó a cabo desde el 21 de octubre de 2012 al 21 de febrero 2013.

El porcentaje de supervivencia de plántulas se calculó mediante la fórmula:

$$
\% \text { supervivencia }=\left(\frac{\text { No. } \text { plántulas supervivientes }}{\text { No. } \text { semillas germinadas }} \times 100\right)[1]
$$


El tiempo medio de germinación (TMG) se calculó mediante la fórmula (Ranal et al. 2009):

$$
\mathrm{TMG}=\frac{\sum\left(n_{i} \times \mathrm{i}\right)}{\mathrm{N}}
$$

Donde, $n_{\mathrm{i}}$ es el número de semillas que germinaron en el día i y $\mathrm{N}$ es el número de semillas germinadas en el período de estudio.

Análisis de datos. Para cada especie se realizó un ANDEVA de una vía para el porcentaje de germinación, supervivencia y el tiempo medio de germinación mediante el procedimiento de modelos lineal generalizados (GLM) con el software de Análisis Estadístico SAS. Se utilizó la prueba de Tukey de comparaciones múltiples, para determinar diferencias significativas entre tratamientos $(\alpha=0,05)$. Los datos de porcentaje fueron transformados previo al análisis mediante la transformación arcsen $(\% \mathrm{G} / 100)^{1 / 2}$ para lograr homocedasticidad y normalidad de los residuos (Ranal et al. 2009). Además se realizó un ANDEVA de dos vías para el porcentaje de germinación y el tiempo medio de germinación, cada uno como variable dependiente y los tratamientos y las especies como independientes, para analizar la interacción entre tratamientos y especies. Las diferencias significativas se evaluaron con Tukey.

\section{RESULTADOS}

Se observó un $2 \%( \pm 2)$ de germinación de semillas de $N$. antarctica en los tratamientos de 100 y $120^{\circ} \mathrm{C}$ y no se registró germinación en el control y en el tratamiento de $80^{\circ} \mathrm{C}$. Por el bajo éxito germinativo de esta especie estos datos no fueron incluidos en los análisis posteriores.

Temperaturas mayores a $100{ }^{\circ} \mathrm{C}$ disminuyeron el porcentaje de germinación de semillas $\left(P_{\text {tratamiento }}<0,001\right)$, además hubo diferencias en la germinación entre especies $\left(P_{\text {especie }}<0,001\right)$ y la interacción tratamientos y especies fue significativa $\left(P_{\text {tratamientoxespecie }}<0,001\right)$ (cuadro 1 ).

La germinación en el control y a $80^{\circ} \mathrm{C}$ de $A$. araucana fue significativamente mayor en relación a la respuesta de germinación de $P$. contorta en todos los tratamientos. Para $P$. con-

Cuadro 1. Diferencias en el porcentaje de germinación de semillas de Araucaria araucana y Pinus contorta bajo diferentes tratamientos de alta temperatura. ANDEVA de dos vías. ${ }^{* * *} P<0,001$.

Seed germination differences in $A$. araucana and $P$. contorta under different high temperatures treatments. Two way ANOVA. ${ }^{* * *} P<0.001$.

\begin{tabular}{lcccl}
\hline & DF & MS & F & \\
\hline Tratamiento & 3 & 1,493 & 39,42 & $* * *$ \\
Especie & 1 & 2,068 & 54,61 & $* * *$ \\
Tratamiento x Especie & 3 & 3,055 & 26,89 & $* * *$ \\
Error & 32 & 0,038 & & \\
\hline
\end{tabular}

torta no hubo diferencias entre el control y los tres tratamientos de alta temperatura. La germinación de P. contorta a 100 y $120{ }^{\circ} \mathrm{C}$ fue significativamente mayor a la germinación de A. araucana en los mismos rangos de temperatura (figura 1A).

En las plántulas de A. araucana se observó mortalidad en el control y en el tratamiento de $80^{\circ} \mathrm{C}$. Los análisis mostraron una disminución significativa $(P<0,001)$ en la supervivencia de plántulas en el tratamiento de $80^{\circ} \mathrm{C}$ (figura 1B). En las plántulas de $P$. contorta no hubo variación en la mortalidad, por lo que al igual que la germinación no se registró cambios en la supervivencia de plántulas con la exposición a diferentes temperaturas (figura $1 \mathrm{C}$ ).

En los tiempos promedios de germinación se realizó un análisis de varianza de dos vías, para evaluar diferencias significativas entre especies mediante la interacción tratamientos y especie, el cual no fue significativo $F(7,32)$ $=2,87, P>0,05$. El análisis de varianza de una vía para cada especie, no mostró diferencias en los tiempos de germinación en los diferentes tratamientos para $P$. contorta, $F(3,16)=0,77, P>0,05$ (cuadro 2). Araucaria araucana mostró diferencias significativas entre los tratamientos que presentaron germinación y en los que se vio inhibida, $F(3,16)=134,15, P<0,0001$ (cuadro 2). En A. araucana el control inició la germinación el día 10, mientras que en el tratamiento de $80^{\circ} \mathrm{C}$ empezó el día 22. Para ambos tratamientos, la máxima germinación se observó entre los días 24 al 47 y la germinación finalizó entre los días 94-99 (figura 2). En P. contorta la germinación para todos los tratamientos inició entre los días 13-15, y el período de máxima germinación se observó entre los días 13 al 24. La mayoría de los tratamientos terminaron de germinar entre los días 72-89 a excepción del control que en el día 115 aún germinó una semilla. En el tratamiento a $80^{\circ} \mathrm{C}$ $P$. contorta mostró una energía germinativa cerca del $15 \%$ a los 25 días, mostrando una estimulación en su germinación respecto a los otros tratamientos (figura 2).

Cuadro 2. Tiempo medio de germinación (TMG) en días (error estándar) para Araucaria araucana y Pinus contorta bajo diferentes tratamientos de alta temperatura. Comparación dentro de la especie. Letras indican diferencias significativas $(P<0,05)$ mediante la prueba de comparación múltiple Tukey.

Mean germination time (MGT) in days (standard error) for $A$. araucana and $P$. contorta under different high temperatures treatments. Comparison within species. Letters a-c indicate significant differences ( $P$ $<0.05$ ) by Tukey's multiple comparison test.

\begin{tabular}{lrrrr}
\hline \multirow{2}{*}{ Tratamiento } & \multicolumn{4}{c}{ TMG (días) } \\
\cline { 2 - 5 } & A. araucana & \multicolumn{1}{c}{ P. contorta } \\
\hline Control & $44,1(3,1)$ & a & $44,1(20,3)$ & a \\
$80^{\circ} \mathrm{C}$ & $42,6(3,0)$ & a & $23,1(4,5)$ & a \\
$100^{\circ} \mathrm{C}$ & 0 & b & $39,7(15,6)$ & a \\
$120^{\circ} \mathrm{C}$ & 0 & b & $17,2(13,8)$ & a \\
\hline
\end{tabular}


A)

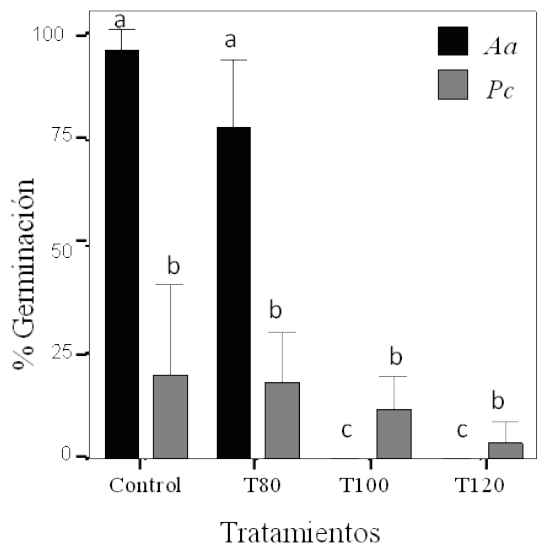

B)

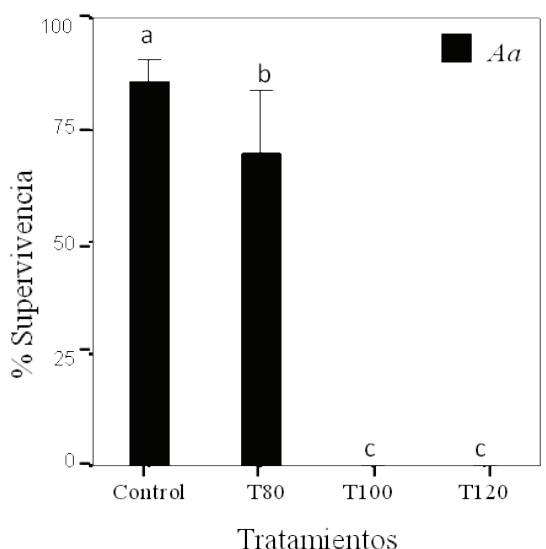

C)

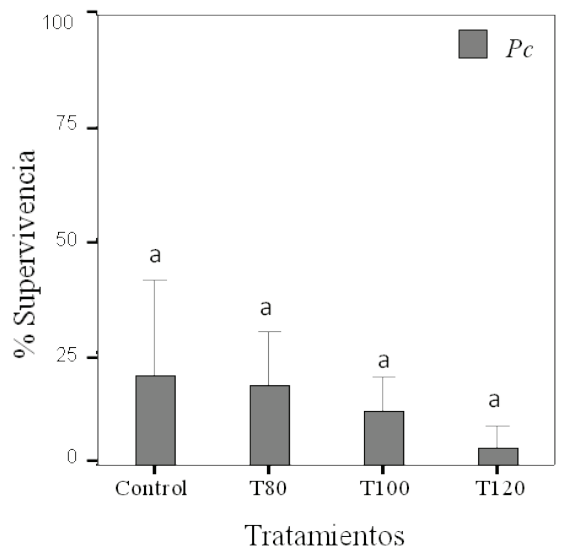

Figura 1. Germinación y supervivencia promedio ( \pm error estándar) de plántulas en los diferentes tratamientos de temperatura. A) Porcentaje de germinación de semillas de A. araucana y P. contorta bajo diferentes tratamientos de altas temperaturas, B) Porcentaje de supervivencia de plántulas al día 126 de $A$. araucana y C) $P$. contorta bajo diferentes tratamientos de altas temperaturas. Letras a-c indican diferencias significativas $(P<0,05)$ entre los tratamientos mediante la prueba de comparación múltiple Tukey. Aa: Araucaria araucana, Pc: Pinus contorta.

Mean germination and seedling survival ( \pm standard error) for the different high temperature treatments. A) Germination percentage of $A$. araucana and $P$. contorta seeds under different high temperature treatments, B) Seedling survival percentage at the day 126 of $A$. araucana and C) $P$. contorta under different high temperature treatments. Letters a-c indicate significant differences $(P<0.05)$ by Tukey's multiple comparison test. Aa: A. araucana, Pc: P. contorta.
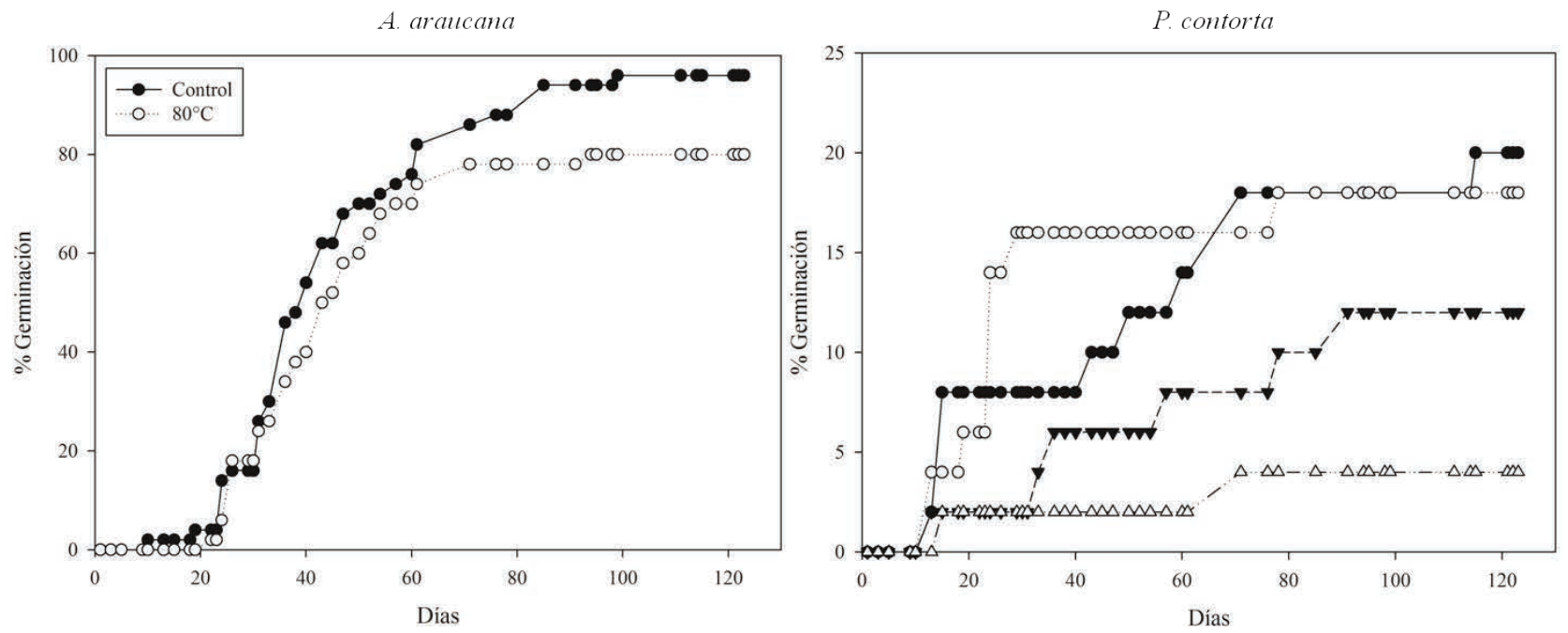

Figura 2. Germinación promedio acumulada de semillas por días para Araucaria araucana y Pinus contorta para los diferentes tratamientos de alta temperatura.

Accumulated mean seed germination by days for A. araucana and P. contorta for different high temperature treatments.

\section{DISCUSIÓN}

Pinus contorta, a pesar de presentar bajos porcentajes de germinación, presenta una ventaja competitiva en comparación a $A$. araucana, ya que sus semillas soportan temperaturas de hasta $120^{\circ} \mathrm{C}$, en comparación a $A$. araucana donde la germinación se ve inhibida a temperaturas superiores a $100{ }^{\circ} \mathrm{C}$. Esto aunado a que la producción anual de semillas de $P$. contorta es mayor a $A$. araucana y que la capacidad germinativa de las semillas de $N$. antarctica es muy baja.

Estudios de $N$. antarctica reportan una capacidad germinativa de $1,7 \%$ con semillas no sometidas a pre-tratamiento y 20,6 \% después de estratificación fría de 90 días (Donoso et al. 2013). Las semillas utilizadas fueron estratificadas, sin embargo, el porcentaje de germinación fue bajo en todos los tratamientos, probablemente esto se 
debe a una baja viabilidad de las semillas, por lo que se recomienda realizar, previo a los ensayos, una prueba de germinación para evaluar la calidad de las mismas. Los bajos resultados de germinación para $N$. antarctica obtenidos en el estudio, concuerdan con lo reportado por otros autores, quienes sugieren que la reproducción sexual no es una estrategia muy importante para la mantención de sus poblaciones, en comparación a la reproducción vegetativa por rebrotes (Bahamonde et al. 2011, Donoso et al. 2013). Incluso, después de incendios forestales se ha observado que la estrategia de regeneración de $N$. antarctica es mediante rebrotes de raíces que sobreviven los incendios (Donoso et al. 2013).

La germinación de $P$. contorta no presentó una diferencia entre el control y los tratamientos de alta temperatura, a pesar de observarse una tendencia a disminuir; resultados que no concuerdan con lo reportado por otros estudios para la misma especie, donde la germinación disminuyó a temperaturas mayores a $75{ }^{\circ} \mathrm{C}$ (Knapp y Anderson 1980, Retamal 2012). No existe una tendencia general del efecto de las altas temperaturas en la capacidad germinativa para otras especies del género Pinus. Por ejemplo, las altas temperaturas disminuyen la capacidad germinativa en Pinus sylvestris L., Pinus halepensis Mill. y Pinus nigra Aiton (Casal 1995, Habrouk et al. 1999), mientras que temperaturas entre $70^{\circ} \mathrm{C}-110{ }^{\circ} \mathrm{C}$ no afectan la germinación en Pinus pinaster Aiton y Pinus radiata D. Don., incluso al compararlo con el control (Casal 1995, Herrero et al. 2007).

Los bajos porcentajes de germinación observados para P. contorta coinciden con los estudios de Casal (1995) para $P$. radiata donde la germinación no supera el $22 \%$ de germinación en el control y a temperaturas de 90 a 110 ${ }^{\circ} \mathrm{C}$ la germinación es de 14,4 y 13,9 \%. De igual forma, estudios de Knapp y Anderson (1980) para P. contorta reportan que la germinación en el control es de 24,8 \% y esta disminuye de 13,3 a 11,3\% a temperatura entre 90 $110^{\circ} \mathrm{C}$. Estos bajos valores de germinación no se observan en otras especies de Pinus spp. Estudios con P. sylvestris, $P$. halepensis y P. nigra, reportan altos porcentajes de germinación (entre 60-90 \%) en semillas sometidas a temperaturas de $70-100{ }^{\circ} \mathrm{C}$ por cinco minutos (Habrouk et al. 1999). Retamal (2012) reporta porcentajes de germinación entre 92-98 \% para P. contorta a temperaturas entre $60-80{ }^{\circ} \mathrm{C}$, resultados que no concuerdan con Knapp y Anderson (1980) y los resultados obtenidos en este estudio.

Estudios con A. araucana reportan una germinación del $60 \%$ a $100{ }^{\circ} \mathrm{C}$ (Boberg et al. 2010), sin embargo, nuestros resultados muestran inhibición de la germinación a 100 y $120^{\circ} \mathrm{C}$. Considerando los resultados reportados por la literatura y los hallazgos de este estudio, se sugiere que $A$. araucana tiene una tendencia a disminuir la germinación al aumentar la temperatura, pero estas diferencias no son significativas para temperaturas medias $\left(50-80^{\circ} \mathrm{C}\right)$, pero temperaturas mayores a $100{ }^{\circ} \mathrm{C}$ muestran inhibición de la germinación.
Las diferencias en los valores de germinación tanto para P. contorta como A. araucana, puede deberse a dos factores: 1) a exceso de riego que disminuyó la germinación de $P$. contorta; en pruebas de germinación en invernadero se determinó una disminución de la germinación por exceso de humedad en el sustrato, recomendándose un contenido de humedad entre 6 y $12 \%$ (Bates 1930); 2) a diferencias metodológicas en el calentamiento de la semilla. La mayoría de estudios donde se evalúa el efecto de altas temperaturas en la germinación se han realizado calentando directamente la semilla, sin embargo, esto no refleja exactamente la realidad en un incendio forestal, ya que el calor se perdería más rápidamente en la semillas de lo que realmente ocurre en el suelo. Se ha determinado que los tiempos de calentamiento del suelo y la forma en que se transfiere el calor son los que causan mayor daño bajo el suelo (DeBano et al. 1998) y en consecuencia al banco de semillas. Odion y Davis (2000) determinaron en un incendio de alta severidad en los matorrales de California, que a una profundidad de $2 \mathrm{~cm}$ los suelos alcanzaron una temperatura máxima de $150{ }^{\circ} \mathrm{C}$ y temperaturas $>100{ }^{\circ} \mathrm{C}$ permanecieron por más de una hora. La máxima temperatura disminuyó rápidamente con la profundidad, alcanzando 51 y $39^{\circ} \mathrm{C}$ a 5 y $10 \mathrm{~cm}$, respectivamente. Esto comprueba que la combinación de la combustión y la transferencia del calor producen gradientes de temperatura en el suelo, temperaturas que disminuyen con la profundidad (DeBano et al. 1998).

Las semillas de $A$. araucana soportan temperaturas medias $\left(50-80{ }^{\circ} \mathrm{C}\right)$ sin afectar su capacidad germinativa y tienen mayores porcentajes de germinación que $P$. contorta a estas temperaturas. Sin embargo, las semillas de $P$. contorta aunque tengan bajos porcentajes de germinación tienen mayor capacidad de soportar altas temperaturas en comparación a A. araucana. Por lo que las semillas de $P$. contorta tendrán mayores posibilidades de sobrevivir a incendios intensos, sin embargo, esto dependerá de la disponibilidad de semillas en el ecosistema. Además, hay que tomar en cuenta que las semillas de A. araucana están sometidas a fuertes presiones de depredación por roedores y la cachaña (E. ferrugineus), y que el rango de dispersión de la semilla es muy bajo (Sanguinetti y Kitzberger 2009), disminuyendo la disponibilidad de las semilla en el banco y limitando su regeneración y establecimiento después de incendios forestales.

Las tres especies de este estudio están adaptadas a regímenes de incendios, y se ha determinado que en incendios de alta intensidad, que provocan la destrucción de los rodales forestales, la recuperación de A. araucana y P. contorta está determinada por la supervivencia de algunos individuos y del banco de semillas aéreo y edáfico (Turner et al. 2007, Teste et al. 2011, González et al. 2013). Además, A. araucana tiene la capacidad de rebrotar (González et al. 2013), mientras que $N$. antarctica depende de la supervivencia de las raíces y posterior rebrote (Donoso et al. 2013). Pinus contorta produce conos abiertos y cerrados, la producción de conos abiertos permite la colonización gradual que ocu- 
rre en bosques con árboles dispersos creando banco de semillas; mientras que los conos serótinos ofrecen un mecanismo de rápido establecimiento post-fuego de plántulas (Turner et al. 2007). Esta capacidad provee a P. contorta una ventaja competitiva en la regeneración sobre $A$. arauca$n a$, aunque, para esta última, se ha observado que los conos pueden tener una función de protección de la semilla a altas temperaturas (González et al. 2013) y están ubicados a mayor altura.

Pinus contorta produce semillas viables a temprana edad, presenta una alta producción de semillas, la dispersión por viento le permite abarcar grandes distancias y las plántulas tienen rápido crecimiento, por lo que representa un buen competidor en el establecimiento tras eventos de perturbación y una especie altamente invasiva (Richardson et al. 1994). Esto sugiere que en la Reserva Nacional Malalcahuello tras un evento de incendios, la invasión de $P$. contorta continuaría y las poblaciones podrían nuevamente establecerse, teniendo una ventaja sobre las especies nativas por poseer semillas con mayor tolerancia a altas temperaturas. Estudios como estos en donde se evalúa la capacidad de respuesta a altas temperaturas de las semillas de especies invasoras son importantes para predecir la posible trayectoria sucesional de ecosistemas invadidos después de incendios forestales y desarrollar oportunamente las estrategias de manejo y restauración necesarias a implementar.

\section{AGRADECIMIENTOS}

Estudio financiado por FONDECYT 1100792, ICM P05- 002 y CONICYT PFB-23. A la beca de la Organización de Estados Americanos (OEA) por el financiamiento de la autora. Agradecimientos especiales a Fernando Contreras del Laboratorio de Semillas y, al Dr. Manuel Sánchez, Decano de la Facultad de Ciencias Forestales por el uso del invernadero, y los revisores anónimos que ayudaron a mejorar este manuscrito. Este estudio se enmarca en el trabajo del Laboratorio de Invasiones Biológicas (LIB), www.lib.udec.cl.

\section{REFERENCIAS}

Bahamonde HA, PL Petri, LH Monelos, L Martínez Pastur. 2013. Aspectos ecológicos de la regeneración por semilla en bosques nativos de Nothofagus antarctica en Patagonia Sur, Argentina. Bosque 32(1): 20-29.

Bates CG. 1930. The production, extraction, and germination of lodgepole pine seed. USDA Tech Bulletin 191: 92.

Boberg P, E Raffaele, E Chaia, J Eneström, L Pettersson, T D'Hertefeldt. 2010. The effect of high temperatures on seed germination of one native and two introduced conifers Patagonia. Nordic Journal of Botany 28: 231-239.

Casal R. 1995. Germination behavior of three species of the genus Pinus in relation to high temperatures suffered during forest fires. Annals of Forest Science 52: 385-392.

Cóbar-Carranza AJ, RA García, A Pauchard, E Peña. 2014. Effect of Pinus contorta invasion on forest fuel properties and its potential implications on the fire regime of Araucaria araucana and Nothofagus antarctica forests. Biological Invasions 16: 2273-2291. DOI 10.1007/s10530-014-0663-8

DeBano LF, DG Neary, PF Folliott. 1998. Fire's Effects on Ecosystems. New York, USA. Wiley. 333 p.

Donoso CZ, L Steinke, A Premoli. 2013. Nothofagus antarctica (G. Forster) Oerst. In Donoso C ed. Las especies arbóreas de los bosques templados de Chile y Argentina: Autoecología. Valdivia, Chile. 2da. Edición. Marisa Cuneo Ediciones. p. $402-410$.

González M, M Cortés, L Gallo, S Bekessy, C Echeverría, F Izquierdo, P Montaldo. 2013. Araucaria araucana (Molina) K. Koch. In Donoso C ed. Las especies arbóreas de los bosques templados de Chile y Argentina: Autoecología. Valdivia, Chile. 2da. Edición. Marisa Cuneo Ediciones. p. 36-53.

Habrouk A, J Retana, JM Espelta. 1999. Role of heat tolerance and cone protection of seeds in the response of three pine species to wildfires. Plant Ecology 145: 91-99.

Herrero C, R San Martin, F Bravo. 2007. Effect of heat and ash treatments on germination of Pinus pinaster and Cistus laurifolius. Journal of Arid Environments 70: 540-548.

Klinka K. 2002. Pinus contorta Douglas ex Loudon. In CAB International ed. Pines of Silvicultural Importance. New York, USA. CABI Publishing. p. 67-78.

Knapp AK, JE Anderson. 1980. Effect of heat on germination of seeds from serotinous lodgepole pine cones. The American Midland Naturalist 104(2): 370-372.

Langdon B, A Pauchard, M Aguayo. 2010. Pinus contorta invasion in the Chilean Patagonia: local patterns in global context. Biological Invasions 12: 3961-3971.

Ledgard N. 2001. The spread of lodgepole pine (Pinus contorta, Dougl.) in New Zeland. Forest Ecology and Management 141: 43-57.

Núñez MA, E Raffaele. 2007. Afforestation causes changes in post-fire regeneration in native shrubland communities of northwestern Patagonia, Argentina. Journal of Vegetation Science 18: 827-834.

Odion DC, FW Davis. 2000. Fire, soil heating, and the formation of vegetation patterns in Chaparral. Ecology Monograph 70(1): 149-169.

Peña E, M Hidalgo, B Langdon, A Pauchard. 2008. Patterns of spread of Pinus contorta Dougl. ex Loud. invasion in a Natural Reserve in southern South America. Forest Ecology and Management 256: 1049-1054.

Ranal MA, D García de Santana, WR Ferreira, C Mendes-Rodríguez. 2009. Calculating germination measurements and organizing spreadsheets. Revista Brasileira de Botanica 32(4): 849-855.

Retamal CJ. 2012. Efecto del fuego y caracterización de la semilla de Pinus contorta. Tesis Licenciado en Biología. Concepción, Chile. Facultad de Ciencias Naturales y Oceanográficas, Universidad de Concepción. 35 p.

Richardson DM, PA Williams, RJ Hobbs. 1994. Pine invasions in the southern hemisphere: Determinants of spread and invadibility. Journal of Biogeography 21: 511-527.

Sanguinetti J, T Kitzberger. 2009. Efectos de la producción de semillas y la heterogeneidad vegetal sobre la supervivencia de semillas y el patrón espacio-temporal de establecimiento de plántulas en Araucaria araucana. Revista Chilena de Historia Natural 82: 319-335. 
Stevens FK. 1996. Germinación de semillas de lenga, coihue de magallanes y ñirre a diferentes temperaturas y regímenes de aplicación. Tesis Ingeniero Forestal. Concepción, Chile. Universidad de Concepción. 79 p.

Teste FP, VJ Lieffers, SM Landhäusser. 2011. Viability of forest floor and canopy seed banks of Pinus contorta var. latifolia (Pinaceae) forests after a mountain pine beetle outbreak. American Journal of Botany 98(4): 630-637.
Turner MG, DM Turner, WH Romme, DB Tinker. 2007. Cone production in young post-fire Pinus contorta stands in Greater Yellowstone (USA). Forest Ecology and Management 242: 119-126.

Zalba SM, YA Cuevas, RM Boó. 2008. Invasion of Pinus halepensis Mill. Following a wildfire in an Argentine grassland nature reserve. Journal of Environmental Management 88: 539-546.

Recibido: 13.02.14

Aceptado: 22.12.14 